\title{
Continuum approach to the numerical simulation of material failure in concrete
}

\author{
J. Oliver* ${ }^{\ddagger}$, A.E. Huespe ${ }^{\S} 2$, E. Samaniego ${ }^{\dagger 1}$, E.W.V. Chaves ${ }^{\dagger 1}$ \\ ${ }^{1}$ E.T.S. Enginyers de Camins, Canals i Ports, \\ Technical University of Catalonia

\begin{abstract}
SUMMARY
Some new aspects of the continuum strong discontinuity approach (CSDA) to model material failure in geomaterials are addressed. A new global algorithm, for tracking multiple crack lines/surfaces in $2 \mathrm{D} / 3 \mathrm{D}$ cases is proposed. It is based on solving a simple heat conduction-like problem accompanying the standard mechanical algorithm. A viscous perturbation method on the crack surface is also proposed to remedy the instabilities caused by mutual interactions of multiple developing cracks. A simple procedure to compute the critical time step that ensures algorithmic uniqueness is then provided. Numerical simulations of two and three dimensional problems displaying a multi-crack pattern are finally presented. Copyright (c) 2000 John Wiley \& Sons, Ltd.
\end{abstract}

KEY WORDS: material failure, fracture, localization, multi-cracking, strong discontinuity.

\section{MOTIVATION}

Material failure in solid mechanics, particularly in geomaterials such as soils, concrete, rocks, etc. is characterized, at the macroscopic level, by the appearance of locus of damage, that exhibit jumps (discontinuities) in the displacement field across them. These discontinuity loci which, depending on the context, are termed, cracks, shear bands, fractures will be from now on referred to as material failure surfaces and the corresponding displacement jumps as strong discontinuities. Continuum modelling of strong discontinuities is classically carried out by inducing concentration of strains in narrow bands (strain localization) in such a way that the

\footnotetext{
${ }^{*}$ Correspondence to: Prof. Javier Oliver

${ }^{\dagger}$ Research assistant

‡Professor: e-mail: oliver@cimne.upc.es

§Researcher from: CIMEC/ CONICET-UNL, Argentina. e-mail: ahuespe@intec.unl.edu.ar

Contract/grant sponsor: Spanish Ministry of Science and Technology; contract/grant number: MAT2001-3863C03-03
}

Copyright (c) 2000 John Wiley \& Sons, Ltd. 
relative displacement at both sides of the localization band stands for the displacement jump. Strain localization has been for a long time a challenging subject in computational mechanics due to the difficulties emerging from the strain softening in the material model that is required in order to induce that localization. The resulting models in continuum approaches, endow the solid response with material and structural instabilities that many times make simulations very cumbersome if not impossible. In addition, the corresponding boundary value problem becomes ill-posed which translates into the well known lack of mesh size and mesh bias objectivity of the corresponding numerical simulations $([3])$.

Enriched continuum based approaches, like non local ([15]) or gradient enhanced models ([30]) provide improved solutions. However, for practical purposes in numerical simulation settings, they require the size of the localization band to be captured by several finite elements ([11]). Then, due to its small bandwidth, in comparison with the structural size, very fine discretizations are required, this leading to many times unbearable computational costs for practical problems.

The inclusion of real discontinuities in the displacement field seems then an appropriate alternative for modeling material failure from a phenomenological standpoint. Classically, this approach has been tightly related to the so-called discrete or cohesive models approaches ([12]). They are based on inserting a discontinuity interface governed by a discrete (cohesive) law relating the traction vector to the crack opening after some local failure criterion is fulfilled. Therefore, two different material laws govern the solid behaviour: a) a continuum (stressstrain) constitutive model for the material bulk and b) a discrete (traction-displacement jump) constitutive model for the discontinuity interface. In consequence, continuum and discontinuum are characterized through different material types and constitutive formats which, in principle, are completely independent from each other.

In this paper an alternative approach, which will be termed the Continuum Strong Discontinuity Approach (CSDA) is tackled. It can be characterized by this two facts:

a) The same (continuum) format for the kinematics, the infinitesimal strain kinematics, is considered both at the continuous bulk and at the failure interface even if the displacements are discontinuous. In some, mathematical, sense the classical functional spaces used to place strain measures are extended to distributions to accommodate the resulting unbounded strains at that failure surface.

b) The same (continuum, i.e. stress-strain) format is adopted for the constitutive model at both the bulk and the failure surface. Even more, the same constitutive model, equipped with strain softening, is adopted for all the particles of the body. Then a local material bifurcation process is responsible for the onset of failure and different loading processes at continuum and discontinuum material points which, eventually, result into different local stress-strain response from the same constitutive model.

The paper addresses the different aspects involved in the modeling of material failure in geomaterials, specifically in concrete, by means of the CSDA. Some of these aspects, such as the theoretical foundations of the CSDA, the material bifurcation analysis, and the formulation of finite elements with embedded discontinuities, are only sketched. Further information about them can be found elsewhere in the literature (see, for instance, [34], [22], [26], [24], [27]). More attention is paid to other aspects that have not been studied in depth previously and that, in the opinion of the authors, are specially relevant in the study of cracking in concrete. The 

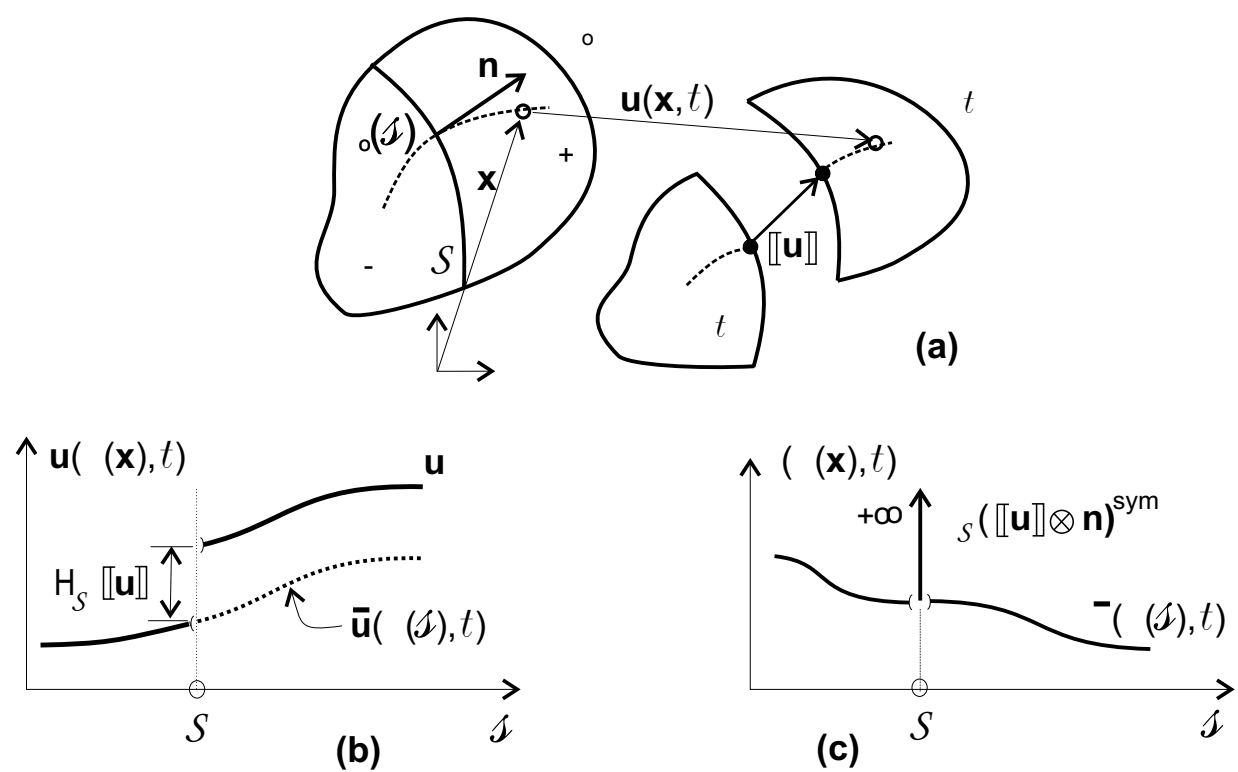

Figure 1. Strong discontinuity in a body

remaining of the paper is organized as follows. In Section 2 the basic ingredients of the CSDA are laid out. Section 3 deals with the criteria to determine the onset and the direction of propagation of a crack path in a consistent way. The finite element technology needed to embed a displacement discontinuity within an element domain is presented in Section 4 . The issue of determining the placement of the crack path within the solid is addressed in Section 5. Section 6 proposes a methodology to circumvent the possibility of loss of uniqueness due to crack arrest-activation related with handling multiple cracks. Finally, the CSDA is applied to the numerical simulation of some problems involving multiple cracks.

\section{THE CONTINUUM STRONG DISCONTINUITY APPROACH (CSDA)}

In the pioneering work from Simo, Oliver and Armero [34] some relevant consequences and requirements from the introduction of strong discontinuities into a continuum medium were identified. Later, the strong discontinuity approach has been further studied and developed in different ways ([22], [1], [25], [17], [27], [28]). In this section we present the basics of that approach faithful to the original spirit based on the combination of the so-called strong discontinuity kinematics (jumps in the displacement field, which give rise to unbounded strains) with the use of continuum constitutive models. In order to achieve this consistently, a crucial ingredient is incorporated: the reinterpretation of the softening modulus in a distributional sense leading to the so-called softening regularization condition. 


\subsection{The strong discontinuity kinematics}

Let us consider the following structure for the displacement field of a body $\Omega$ undergoing a strong discontinuity on the failure surface (crack) $\mathcal{S}$ (see figure: 1):

$$
\mathbf{u}(\mathbf{x}, t)=\overline{\mathbf{u}}(\mathbf{x}, t)+H_{\mathcal{S}}(\mathbf{x}) \llbracket \mathbf{u} \rrbracket(\mathbf{x}, t)
$$

where $H_{\mathcal{S}}$ is the Heaviside's step function, $\llbracket \mathbf{u} \rrbracket$ stands for the jump in the displacement field across $\mathcal{S}$, and $\overline{\mathbf{u}}$ is a continuous displacement field.

Now, the corresponding strain field can be computed as the symmetric gradient of equation (1), yielding

$$
\boldsymbol{\varepsilon}=\boldsymbol{\nabla}^{s} \mathbf{u}=\underbrace{\boldsymbol{\nabla}^{s} \overline{\mathbf{u}}+H_{\mathcal{S}} \boldsymbol{\nabla}^{s} \llbracket \mathbf{u} \rrbracket}_{\overline{\boldsymbol{\varepsilon}} \text { (regular) }}+\underbrace{\delta_{\mathcal{S}}(\llbracket \mathbf{u} \rrbracket \otimes \mathbf{n})^{s}}_{\llbracket \varepsilon \rrbracket(\text { singular })}
$$

where $\delta_{\mathcal{S}}$ is the Dirac's delta function acting on $\Omega$ and $\mathbf{n}$ is the unit normal to $\mathcal{S}$.

For computational purposes (numerical evaluation), a more convenient regularized format for equation (2) is

$$
\varepsilon=\underbrace{\bar{\varepsilon}}_{\text {bounded }}+\underbrace{\mu_{\mathcal{S}} \frac{1}{h}(\llbracket \dot{\mathbf{u}} \rrbracket \otimes \mathbf{n})^{s}}_{\text {unbounded as } h \rightarrow 0}
$$

$\mu_{\mathcal{S}}$ being a collocation function on $\mathcal{S}_{h}\left(\mu_{\mathcal{S}_{h}}=1 \forall \mathbf{x} \in \mathcal{S}\right.$ and $\mu_{\mathcal{S}}=0$ otherwise) and $h$ a regularization parameter. The second term on the right hand side of equation (3) is the regularized version of the singular term in equation (2).

Remark 1. : Expression (3) corresponds to the so-called regularized strong discontinuity kinematics ([34], [16]). At this stage it is emphasized that $h$ is a (mathematical) regularization parameter and not a material property, which should be taken as a number as small as allowed by the machine precision.

\subsection{Continuum constitutive modeling}

Modeling the behavior of geomaterials from a Continuum Mechanics standpoint, requires the consideration of a constitutive model relating stresses and strains, typically:

$$
\sigma=\Sigma(\varepsilon, \mathcal{H})
$$

with $\mathcal{H}$ being the hardening/softening modulus.

Within this context from now on we are being concerned with constitutive models equipped with strain softening. These models are characterized by the negative character of the continuum softening modulus, i.e., $\mathcal{H}<0$.

For the case of rate independent materials, the rate version of equation (4) reads

$$
\dot{\boldsymbol{\sigma}}=\mathbf{C}^{t g}: \dot{\varepsilon}
$$

where $\mathbf{C}^{t g}$ is the so-called constitutive tangent operator. 


\subsection{The distributional character of the softening modulus}

In order to assure compatibility of the strong discontinuity kinematics presented in Section 2.1 with the continuum constitutive models, the continuum softening modulus has to be reinterpreted in a distributional sense ([34], [26]):

$$
\mathcal{H}^{-1}=\delta_{\mathcal{S}} \overline{\mathcal{H}}^{-1}
$$

where $\overline{\mathcal{H}}$ is the intrinsic or discrete softening modulus which is assumed to be a regular distribution (i.e., it is assumed to be bounded) and given in terms of the fracturing properties of the material. If linear softening is modeled, then:

$$
\overline{\mathcal{H}}=\frac{1}{2} \frac{\sigma_{y}^{2}}{G_{f}}
$$

where $\sigma_{y}$ stands for the uniaxial peak strength and $G_{f}$ for the fracture energy.

The regularized version of equation (6) reads

$$
\frac{1}{\mathcal{H}}=\frac{1}{h \overline{\mathcal{H}}} \Longrightarrow \mathcal{H}=h \overline{\mathcal{H}}
$$

\subsection{Some relevant consequences of the CSDA}

The basic ingredients of the CSDA just presented lead to the following crucial results (see [24] for their rigorous derivation):

- As a consequence of expression (6), the boundedness of the stresses $\boldsymbol{\sigma}$ is assured, despite the unbounded character of the strains $\varepsilon$ at $\mathcal{S}$.

- A discrete (cohesive) constitutive law, relating the traction vector, $\mathcal{T}=\boldsymbol{\sigma} \cdot \mathbf{n}$, with the displacement jump $\llbracket \mathbf{u} \rrbracket$, is automatically fulfilled at material points of $\mathcal{S}$. This discrete law can be regarded as a projection of the standard continuum constitutive law into the discontinuity interface. A general expression of these projected constitutive laws reads:

$$
\mathcal{T}=\digamma(\llbracket \mathbf{u} \rrbracket, \overline{\mathcal{H}})
$$

The discrete constitutive law (9) establishes a clear link between the CSDA and the discrete, fracture mechanics based, approaches ( [27]).

\section{ONSET AND PROPAGATION OF A CRACK}

As mentioned above, material failure in a classical continuum setting is related with constitutive models equipped with strain softening. It is well known that the presence of strain softening in rate independent constitutive models can lead to strain localization through a local material bifurcation procedure ( [38]). A necessary condition for strain localization is the singularity of the so-called localization tensor $\mathbf{Q}^{\text {loc }}$ i.e.:

$$
\operatorname{det}\left[\mathbf{Q}^{l o c}\right]=0
$$

where $\mathbf{Q}^{l o c}$ is defined as

$$
\mathbf{Q}^{l o c}=\mathbf{n} \cdot \mathbf{C}^{t g} \cdot \mathbf{n}
$$


where $\mathbf{n}$ is the normal to the localization band.

Thus, a material point $\mathbf{x}$ is said to undergo material bifurcation when condition (10) holds for that point. That particular time at which material bifurcation occurs for a given particle is called bifurcation time.

\section{FINITE ELEMENT TECHNOLOGY}

Discretization of boundary value problems including strong discontinuities by means of the finite element method requires the use of non standard formulations. In order to get a resolution of the displacement jumps in an efficient way, the necessity of embedding discontinuities within an element domain becomes apparent. The jumps in the displacement field can be added to the standard finite element approximation functions as some kind of enriching modes. Here, we classify these enriching techniques into two general types depending on the support of the enriching modes: nodal enrichment and elemental enrichment.

\subsection{Nodal enrichment}

Nodal enrichment is many times based on the partition of unity concept. Applications to linear fracture mechanics can be found in ([18], [5]) and applications to discrete constitutive models can be found in ([37]).

For this kind of enrichment the discontinuities in the approximation function are included in a nodal basis, i.e., the enrichment is associated to a given node. As a consequence, the enrichment affects all the elements belonging to the support of that node and additional degrees of freedom have to be defined for each enriched node (see figure: 2).

The principal advantages of this type of enrichment is that it leads to symmetric matrices and that continuity of the displacement jump function, $\llbracket \mathbf{u} \rrbracket$, along the discontinuity interface is achieved $([14])$. On the other hand, it also implies a high implementation effort and substantial increments in the computational cost due to the additional degrees of freedom.

\subsection{Elemental enrichment}

The enriching modes have an elemental support and act only on those elements crossed by the crack path (figure: 2). The added modes are, therefore, statically condensable at the element level, which is a crucial advantage from the computational cost standpoint. In $2 \mathrm{D}$ analyzes, for instance, there are two additional modes per element (scaled by the two components of the elemental displacement jump, which are two additional elemental degrees of freedom), and the displacement jump is element-wise constant. In [13] and [28] systematic studies of different classes of elemental enrichment were presented and the so-called non-symmetric formulation ([23]) is shown to have a good performance.

\section{ALGORITHMS FOR TRACKING THE CRACK PATH}

Construction of the enriching modes, for the finite elements with embedded discontinuities described in section 4, requires determination of the position of the failure surface inside every element crossed by the crack path. In addition, the crack path should be continuous 
Nodal enrichment

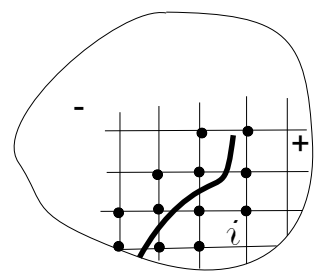

Elemental enrichment

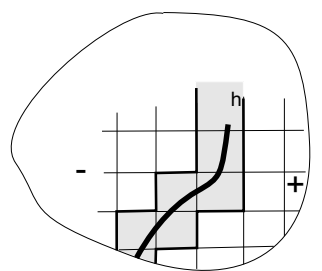

\section{Enriching modes}

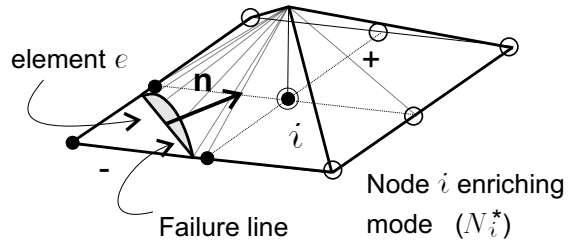

(b)

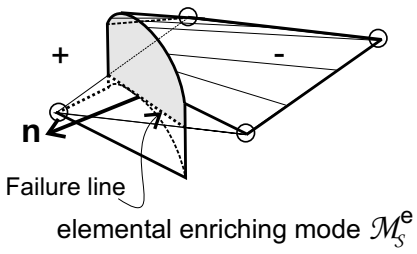

Figure 2. Finite elements with embedded discontinuities

when passing from one element to another. This is done by means of the so called tracking algorithms.

A typical tracking algorithm requires the following information:

1. A failure criterion: it indicates when an element (or some point in it) has undergone material bifurcation and that it has to be added to the set of elements crossed by the discontinuity. A rigorous criterion, in a classical continuum mechanics setting, is the one stated by equation (10). However, other simplified failure criteria, like the ones based on exceeding the peak stress in the principal stress directions, provide enough accurate results for some geomaterials as concrete .

2. A direction of propagation: the orientation of the crack within an element is determined by the propagation direction provided by the normal to the failure surface $\mathbf{n}$. Again, it can be obtained from the discontinuous bifurcation analysis in equation (10), or by the condition $\mathbf{n}=\arg \left\{\min _{\mathbf{n}} \operatorname{det} \mathbf{Q}(\mathrm{n})\right\}$ in the elastic range. For concrete it is frequently taken, from simplified assumptions, as the maximum principal stress direction.

Tracking algorithms can be classified into two groups ${ }^{\dagger}$ as done in [29]: local (or propagation) algorithms and global algorithms.

\footnotetext{
${ }^{\dagger}$ A third type of tracking algorithms is constituted by the ones based on the level set concept ([36]) which share some of the characteristics of local and global algorithms.
} 


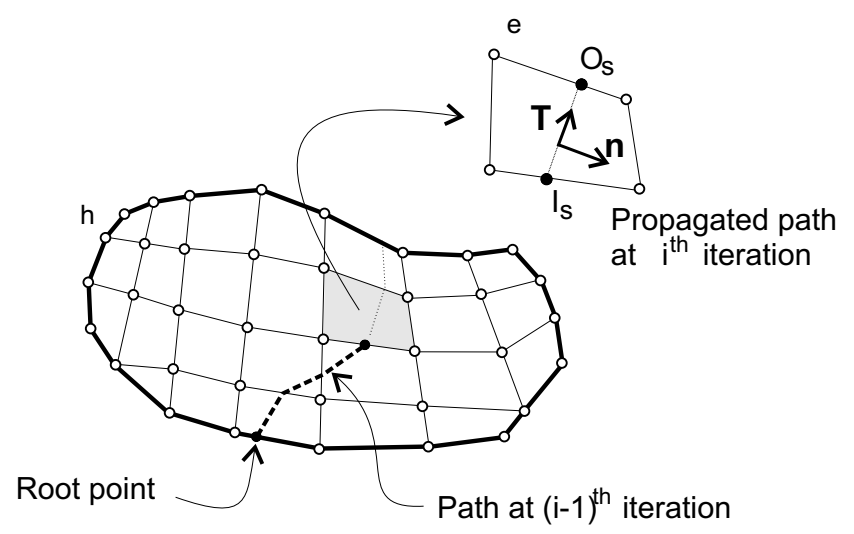

Figure 3. Local propagation algorithm

\subsection{Propagation algorithms}

These algorithms are based on the geometrical propagation of the crack from some root element, which is identified as the first that fulfills the failure criterion, by using point by point local information about the propagation direction. As a matter of example let us consider the following 2-D propagation algorithm:

At every step of the procedure we assume that the following input data is available at the element level:

- A point in one of the sides of the element (which is termed the input side) denoted by $I_{S}$.

- The direction of propagation of the crack in that element, defined by a vector $\mathbf{T}$ (orthogonal to $\mathbf{n}$ ).

The algorithm typically consists of applying the following steps (see figure: 3 ) :

1. Trace a straight line in the direction of $\mathbf{T}$ passing through $I_{S}$.

2. Determine what side of the element, other than the input side, is intersected by the traced line. It will be referred to as output side.

3. Determine the point at which the traced line intersects the output side. It will be denoted by $O_{S}$.

4. Set the output side and the output point $O_{S}$ of the current element as the input side and the input point $I_{S}$ of the neighbor element.

This algorithm has to be recursively applied to all the elements crossed by a crack line.

Geometrically this is nothing else that a Runge-Kutta like algorithm to plot a function $y(x)$ in the $x-y$ space once a point of the curve (the root element) is known and the derivative $y^{\prime}(x)$ (the propagation direction $\mathbf{T}$ ) is point-wise known. Observe that the algorithm requires an element-sides connectivity array in the step 4 above, whose construction involves the access to upper levels of a finite element code. 
Although they are simple and easy to implement for just-one-crack cases, the extension of propagation algorithms to the case of multiple cracks simultaneously developing and interacting with each other is rather cumbersome and can increase significantly the implementation effort. Also the extension to 3D cases, through plane surfaces inside every element, can not be done for general cases $([9])$.

\subsection{Global tracking}

In this type of tracking algorithms, all the possible crack paths are traced at once, on the basis of the information provided by the global propagation field $\mathbf{T}(\mathbf{x}, t) \forall \mathbf{x} \in \boldsymbol{\Omega}$. One can define the crack path candidates at time $t$ as that family of lines which are the envelopes of the propagation vector field $\mathbf{T}(\mathbf{x}, t)$ (tangent to $\mathbf{T}$ at every point $\mathbf{x}$ ) or, equivalently, orthogonal to the normal field $\mathbf{n}(\mathbf{x}, t)$ (see figure: 4 -a). This concept is then immediately extendable to $3 \mathrm{D}$ cases, in terms of those surfaces orthogonal to the normal field $\mathbf{n}(\mathbf{x}, t)$ obtained, for instance, from the propagation criterion (10). Availability of the envelopes of the propagation vector field as crack path candidates allows to manage both the elemental constitutive behaviour and the elemental enriching models on the basis of the following premises:

- From the crack path candidates, a set of active cracks and the corresponding elemental counterparts are selected.

- Among the active elemental crack-paths some are declared consolidated (the element to which they belong has failed) and the remaining are declared potential (where the failure criteria has not yet been reached).

- Only for those elements crossed by consolidated crack paths the enriching discontinuity modes are activated.

- Only those elements crossed by an active crack path (either consolidated or potential) are allowed to soften. The remaining ones are forced to either unload or to harden in all their sampling points.

The previous information is generated through the following algorithm:

1. At the current time $t_{n}$ every element is checked from being crossed by any of the currently active crack paths. If a crossed element had failed for the first time at time $t_{n-1}$ the corresponding elemental crack path is declared consolidated for the rest of the analysis. Otherwise it is declared potential.

2. If an element that had failed at time $t_{n-1}$ is not crossed by any of the currently active crack paths, the element is declared a root element and a new crack is activated. The new crack path is the corresponding envelope of the propagation vector field passing through the centroid of the new root element.

5.2.1. Tracing the envelopes of the propagation vector field The global tracking methodology presented in section 5.2 requires the determination of 1 ) the envelopes (lines in $2 \mathrm{D}$ cases and surfaces in 3D problems) of the propagation vector field and 2) the elements which are crossed by any of those lines or surfaces.

A procedure to trace the envelopes of the propagation vector field is presented here. It is based on finding a scalar vector field $\theta(\mathbf{x})$ whose iso-level curves in 2-D, or iso-level surfaces in $3-\mathrm{D}$, are those envelopes, i.e.,

$$
S_{i}:=\left\{\mathbf{x} \in \boldsymbol{\Omega} ; \theta(\mathbf{x})=\theta_{S_{i}}\right\}
$$



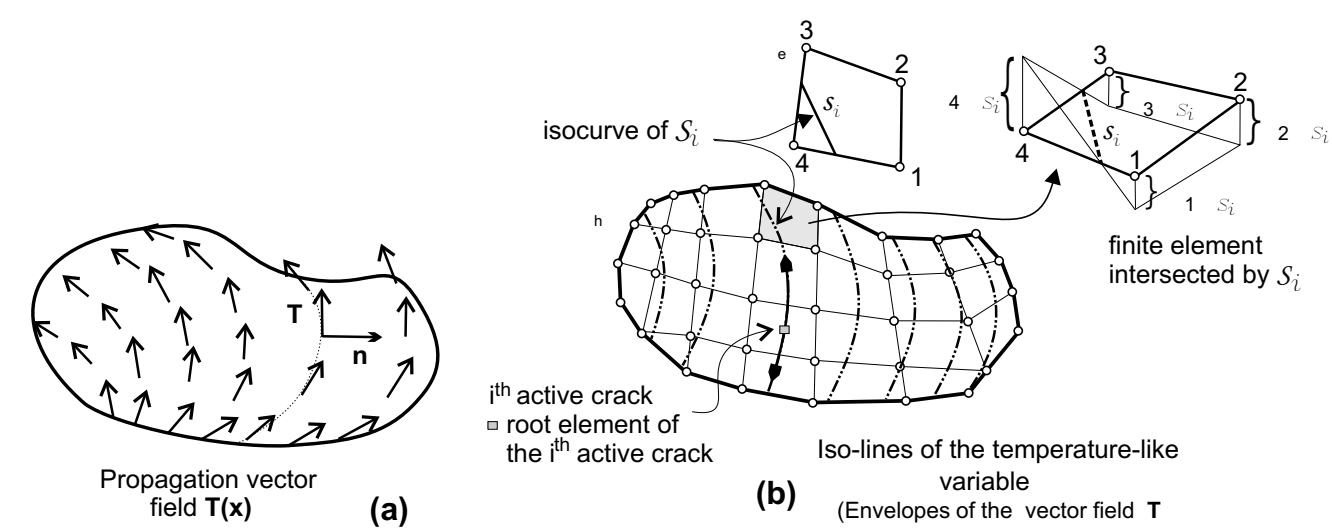

Figure 4. Global propagation algorithm.

where $\theta_{S_{i}}$ is a constant that acts as the label of the $i$-th envelope $S_{i}$. The mathematical condition that has to be fulfilled by such a scalar field $\theta(\mathbf{x})$ are:

$$
\begin{array}{ll}
\frac{\partial \theta}{\partial T}=0 & \quad(\text { for } 2 \mathrm{D} \text { cases }) \\
\frac{\partial \theta}{\partial T}=\frac{\partial \theta}{\partial S}=0 & \quad(\text { for } 3 \mathrm{D} \text { cases })
\end{array}
$$

where $\mathbf{S}$ and $\mathbf{T}$ are any two unit vectors that span the failure plane (orthogonal to $\mathbf{n}$ ), in the three-dimensional case A numerical procedure to solve the PDE's (13), was proposed in [29]. The key idea is to solve the following simplified heat-conduction-like problem with adiabatic heat flux conditions at the boundary $\partial \Omega$ and no internal heat source:

$$
\begin{aligned}
& \text { FIND : } \theta(\mathbf{x}) \\
& \text { SUCH THAT } \\
& \begin{array}{lll}
\boldsymbol{\nabla} \cdot \mathbf{q}=0 & \text { in } \Omega & (a) \\
\mathbf{q}=-\mathbb{K} \cdot \boldsymbol{\nabla} \theta=-\mathbf{T} \frac{\partial \theta}{\partial T}-\mathbf{S} \frac{\partial \theta}{\partial S} & \text { in } \Omega & (b) \\
q_{\nu} \equiv \mathbf{q} \cdot \boldsymbol{\nu}=-(\boldsymbol{\nu} \cdot \mathbf{T}) \frac{\partial \theta}{\partial T}-(\boldsymbol{\nu} \cdot \mathbf{S}) \frac{\partial \theta}{\partial S}=0 & \text { on } \partial \Omega & (c)
\end{array}
\end{aligned}
$$

where $\mathbf{q}$ stands for the heat flux and $\mathbb{K}$ is an anisotropic conductivity-like tensor defined as:

$$
\mathbb{K}=\mathbf{T} \otimes \mathbf{T}+\mathbf{S} \otimes \mathbf{S}
$$

In principle, the singular character of $\mathbb{K}$ can be source of ill-posedness. In order to overcome this singularity, eq. (15) could be modified as:

$$
\mathbb{K}^{\epsilon}=\mathbf{T} \otimes \mathbf{T}+\mathbf{S} \otimes \mathbf{S}+\epsilon \mathbf{1}
$$

where $\epsilon$ is a small perturbation parameter and $\mathbf{1}$ is the unit tensor. However, for practical purposes, the spatial discretization produce enough numerical diffusivity so that the singularity of $\mathbb{K}$ has only been detected in very specific finite element meshes, when the elements are aligned with the $\theta$ iso-curves. Only for that cases it is necessary to introduce the $\epsilon$-perturbation.

For 2-D problems $\mathbf{S}$ is removed from equations (14) and (15). It can be proven ([29]) that the solution of problem (14) is $\mathbf{q}=\mathbf{0}$ and, therefore, from equation (14)-(b), equations (13) are 
fulfilled by the temperature field solution of problem (14). Therefore, the isothermal surfaces (12) of that thermal problem supply the intended envelopes of the propagation vector fields $\mathbf{S}$ and $\mathbf{T}$.

The finite element discretization ${ }^{\ddagger}$ of the BVP (14) yields, after standard procedures ([39]) the following system of linear algebraic equations:

$$
\left[\mathbf{K}_{\theta}^{\epsilon}\right]\{\boldsymbol{\theta}\}=\{\mathbf{0}\}
$$

where $\left[\mathbf{K}_{\theta}^{\epsilon}\right]$ is the "stiffness matrix" of the heat-conduction-like finite element problem and $\{\boldsymbol{\theta}\}$ is the vector of temperature-like nodal unknowns. The rank of the global matrix $\mathbf{K}_{\theta}^{\epsilon}$ is $\left(n_{\text {node }}-1\right)$ where $n_{\text {node }}$ is the number of nodes of the finite element mesh. Thus, the temperature-like variable, should be prescribed, at least, in two nodes, in order to provide a unique non uniform $\theta(\mathbf{x})$ solution, analogously to stationary heat transfer problems where Newman type boundary conditions are imposed. The values of the prescribed temperatures are not relevant whenever they are not prescribed on the same thermal contour.

The thermal problem (17), that can be solved once every time step or every several time steps, is linear and involves only one degree of freedom per element. The resulting computational cost is, therefore, very small. As for the implementation, it is trivial for codes dealing with coupled multifield problems. Otherwise, and since the thermal problem is fairly simplified (adiabatic boundary, no heat source etc.) the implementation is straight-forward.

After solving the system of equations (17) for $\{\boldsymbol{\theta}\}$, the values of the temperature-like variable $\theta$ are known at the nodes and the position of a crack can be determined on an element by element basis by comparison with the reference temperature $\theta_{S_{i}}$ associated to the i-th active crack $S_{i}$ (see figure: 4 -b). Furthermore, the topological problems that a propagation algorithm entails for $3-\mathrm{D}$ problems are readily circumvented.

\subsection{Representative examples from elastic analyzes}

For monotonic loading cases, and from the authors' experience, the vector field $\mathbf{n}(\mathbf{x}, t)$ and, therefore, the propagation vectors fields $\mathbf{S}$ and $\mathbf{T}$ in equations (13)-(14) do not change very much along time. This suggests that, whenever a root element is guessed, the failure (crack) surface can be approximately determined at early stages of the simulation process. Even more, an initial linear elastic analysis and the corresponding stress field $\boldsymbol{\sigma}(\mathbf{x}, 0)$ might be enough to provide an approximated vector field $\mathbf{n}(\mathbf{x}, 0)$ and to determine the failure surface via the thermal-like analysis presented in the previous section. The following two examples correspond to the determination of the failure surface in typical 3D problems from a simple elastic analysis taking the first principal direction as the failure direction $\mathbf{n}[9]$.

- Case a: Anchorage structure. This example was solved in [31] using 2D axisymmetric simulation. Here the solution corresponds to an actual 3D elastic simulation. It consists of an anchorage structure made of concrete in which a bolt is embedded and anchored as shown in figure: 5-a. Assuming that the root lies at the outer part of the anchor disk, the resulting failure surface captured by the heat conduction tracking algorithm is

\footnotetext{
¥This discretization can be performed on the same mesh and the same basic finite element than for the mechanical problem. Thus, it does not involve any new meshing procedure.
} 

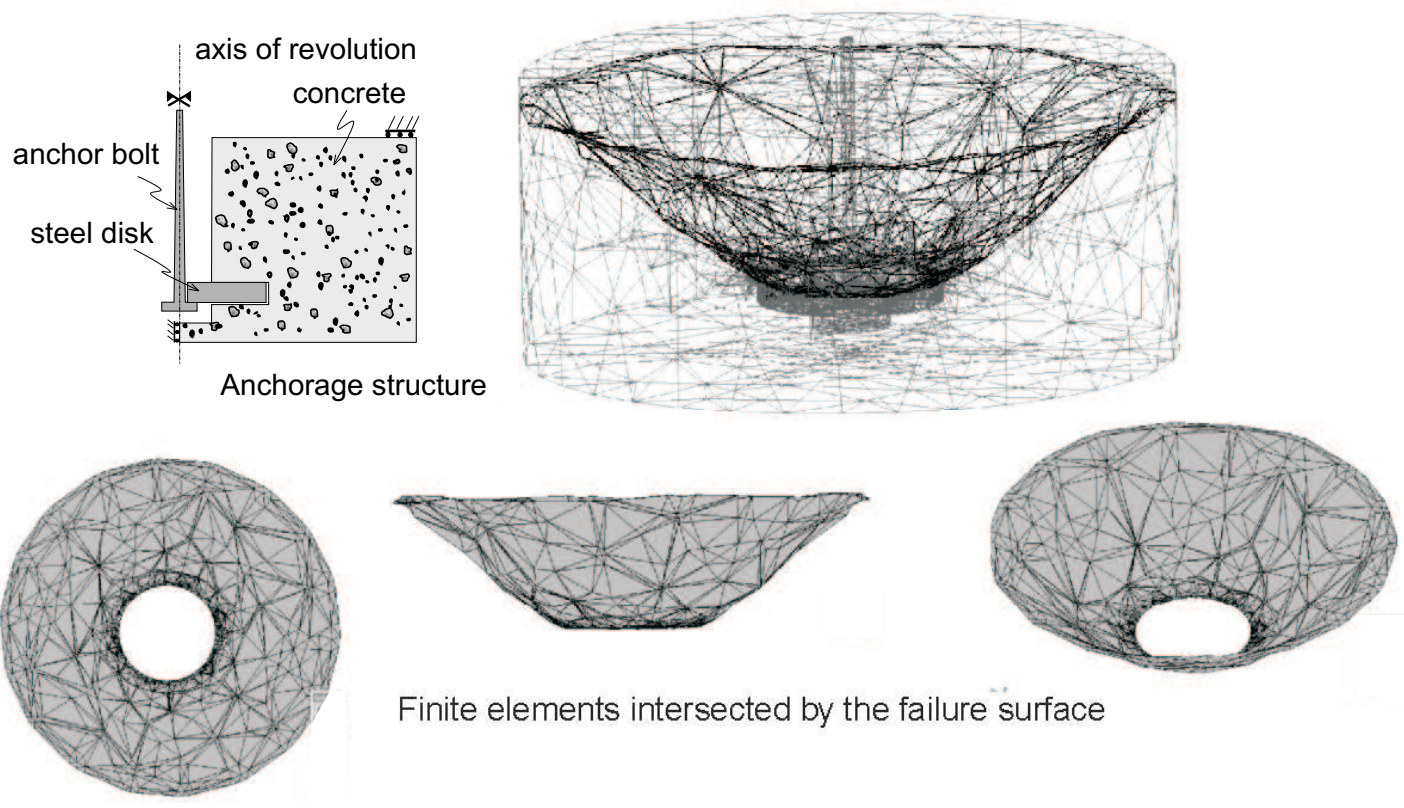

Finite elements intersected by the failure surface

Figure 5. Discontinuity failure surfaces en 3D problems: anchorage structure

presented in figure: 5 . As it can be checked there, it exhibits the typical conical shape experimentally observed in pull out tests on concrete specimens.

- Case b: Double notched shear beam. This test was performed by Bocca et al. [6] who experimentally observed the two cracking surfaces emerging from the notch tips in figure: 6. Some geometrical features and boundary data are shown there. In the figure, two failure surfaces provided by the heat conduction tracking algorithm are presented. It can be observed the close correspondence in trace and shape with the experimentally obtained crack. It is noticeable the high geometrical complexity of the resulting surface, made of hundreds of plane facets obtained by the algorithm.

\section{MULTI-CRACKING TREATMENT}

Consideration of multiple cracks introduces additional complexity in the mathematical and numerical modeling of material failure. The problems and possible remedies are considered in this section.

Physically, cracks can be classified, at least roughly, into two groups:

- Primary cracks: they grow during a relevant part of the deformation process and, therefore, contribute substantially to the global dissipation in the structure. 


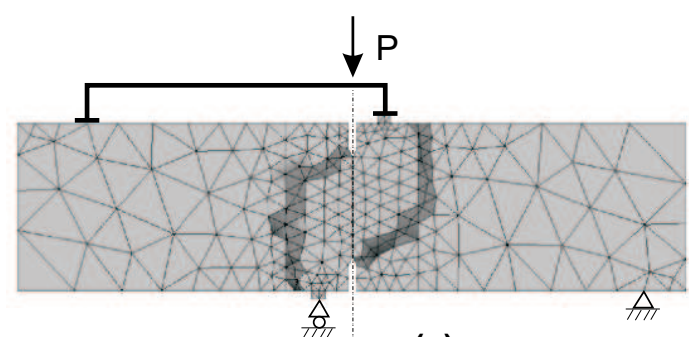

(a)

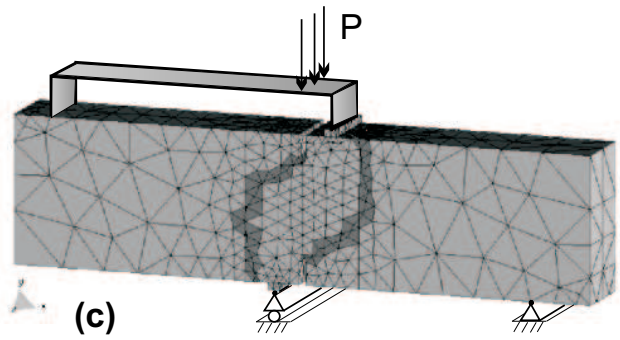

(c)

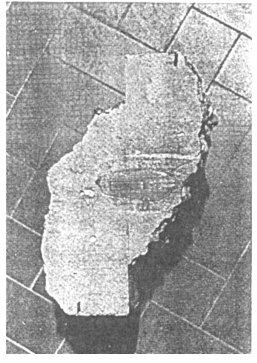

(b) (d)

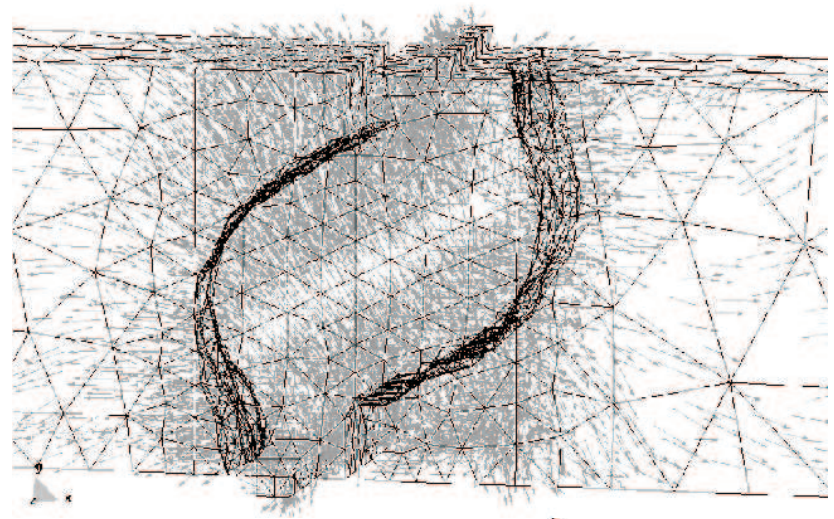

(e)

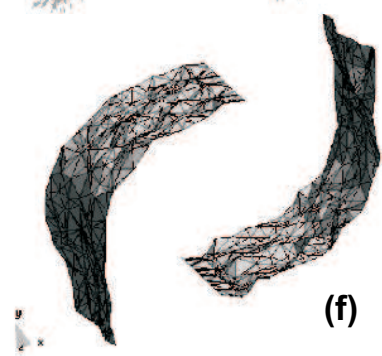

Figure 6. Discontinuity failure surfaces for 3D problems: double notched shear beam. a) and b) experimental crack pattern (from [6]), c) Finite element mesh, d) First principal stress field, e) Failure surfaces in the finite element mesh (the elements crossed by the failure surfaces have been removed for representation purposes) f) Close view of the obtained failure surfaces

- Secondary cracks: they are active just sporadically and are negligible regarding the global dissipation of the structure.

Even in the case of neglecting the effect of secondary cracking (which is not trivial since, in general, whether a crack is primary or secondary is not known in advance) the interaction between primary cracks can lead to difficulties when tracing the overall response of a structure. This is caused by the existence of singular points (bifurcations) on the equilibrium path in the solution space, which result from the interaction between different cracks and their possible combination of activation or arresting states. In this case, not even the use of sophisticated continuation techniques is enough for a successful tracing of the overall response. 


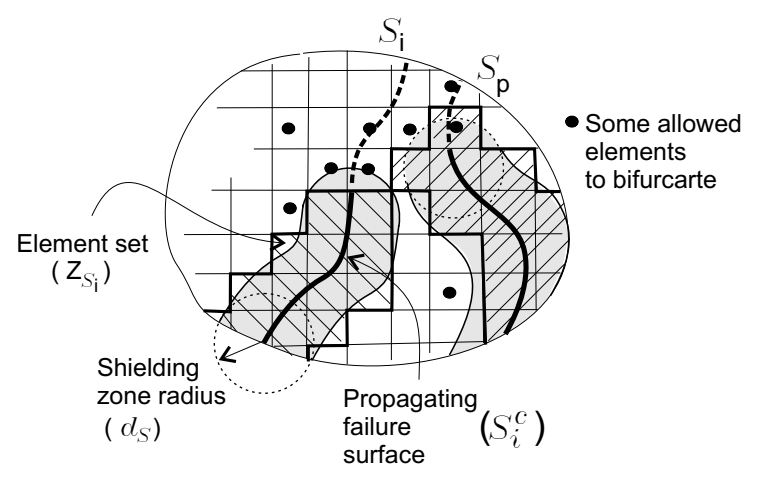

Figure 7. Shielding zone

Despite the fact that the mathematical description leads to bifurcation, it is experimentally observed that only some of the possible branches of the equilibrium path are actually preferred at late stages in the deformation process. From the physical point of view, one expects this preferred branch to be the most stable one. In the case of adiabatic deformation processes this corresponds to the solution yielding the maximum second order work for load control and the minimum second order work for displacement control (see Bazant et al. [4]).

Two complementary ingredients in this approach are designed to prevent this type of similarities and to increase computational robustness. Both of them are described in the following.

\subsection{Shielding}

A neighborhood of a consolidated crack path is defined (the shielding zone) in such a way that the onset of a new crack or the propagation of a different previous consolidated crack path are forbidden.

In a more formal description: let the distance between elements $j$ and $k$ be $d_{j k}=\left\|\boldsymbol{\xi}_{j}^{0}-\boldsymbol{\xi}_{k}^{0}\right\|$ where $\boldsymbol{\xi}^{0}$ are the elemental centroid positions. Consider, also, the $i^{\text {th }}$ failure surface $\mathcal{S}_{i}$ (see figure 7). One part of it, denoted by $\mathcal{S}_{i}^{c}$, is consolidated as defined in section 5.2. Let $\mathcal{J}_{i}$ and $\mathcal{J}_{i}^{c}$ the sets of elements crossed by $\mathcal{S}_{i}$ and $\mathcal{S}_{i}^{c}$, respectively. The shielding zone radius $d_{S}$ determines the set of elements $Z_{\mathcal{S}_{i}}$ belonging to the shielding zone of $\mathcal{S}_{i}^{c}$ as:

$$
Z_{\mathcal{S}_{i}}=\left\{e \in\left\{1, . . n_{\text {elem }}\right\} ; \min _{k \in \mathcal{J}_{i}^{c}} d_{e k} \leq d_{\mathcal{S}}\right\}
$$

At a given time step, an element $e$ will only be allowed to fail if at least one of the following conditions are fulfilled at that time: 1) the element does not belong to the shielding zone of any active crack $\left(e \notin Z_{\mathcal{S}_{i}} \forall i\right)$ or 2$)$ the element belongs to a still not consolidated part of a crack $\left(e \in \mathcal{J} \backslash \mathcal{J}_{i}^{c}\right)$.

Typically, $d_{S}$ takes a value corresponding to the element size. In such a way that the propagation of two cracks through the same element is precluded. 


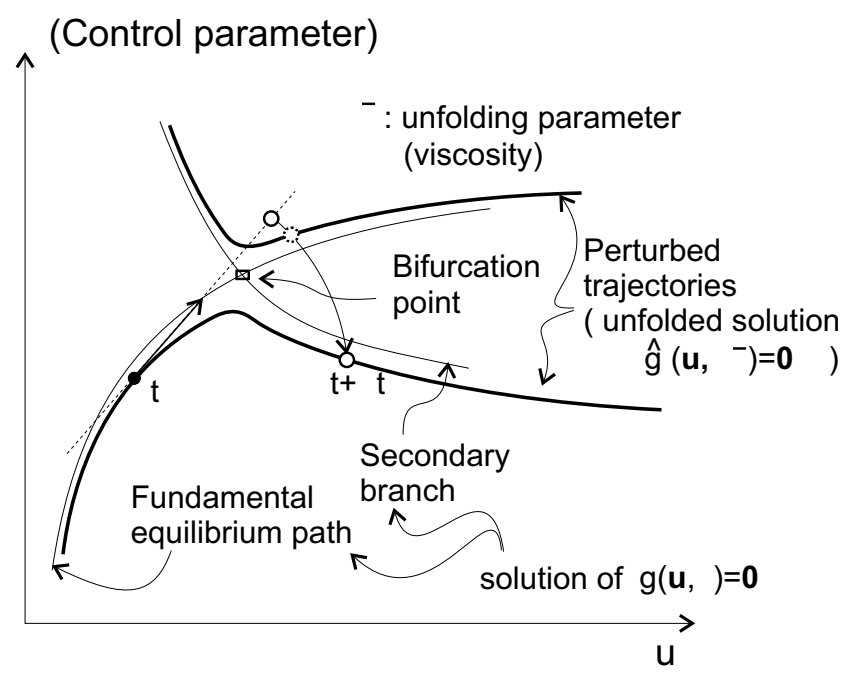

Figure 8. Unfolding a singular trajectory

\subsection{Bifurcation unfolding: Viscous perturbation}

As suggested above, the selection of the correct branch in the presence of a bifurcation point in the equilibrium space entails, in principle, a bifurcation analysis which is not an easy task since it requires resorting to costly branch-switching methods ([21]). A less expensive alternative is to perturb the original problem in order to unfold the bifurcation (see figure: 8 ) such that, in this way, the equilibrium path becomes unique ([21]). Certainly, the problem of determining the perturbation that yields the correct (the most stable) solution is not trivial and would require resorting to some kind of perturbation sensitivity analysis. In this sense, it is also possible to propose a perturbation having a physical meaning that helps to understand the effects that it will entail. Then, its performance with respect to experimental information and to what is physically expectable has to be tested by means of numerical simulations.

Here a viscous perturbation acting at the discontinuity interface in order to achieve a correct unfolding of the equilibrium path is proposed. As a consequence some important uniqueness results are obtained as it is shown in next sections.

6.2.1. Variational Formulation Let us state the following variational boundary value problem (VBVP) corresponding to a solid $\Omega$ (whose boundary is denoted by $\partial \Omega$ consisting of the disjoint subsets $\Gamma_{\boldsymbol{\sigma}}$ and $\Gamma_{u}$, where tractions and displacements are, respectively, prescribed) with an internal discontinuity interface $\mathcal{S}$ (see figure: 9 ):

$$
\int_{\Omega} \nabla^{S} \boldsymbol{\eta}: \boldsymbol{\sigma} d \Omega=\int_{\Omega} \mathbf{b} \cdot \boldsymbol{\eta} d \Omega+\int_{\Gamma_{\sigma}} \mathbf{t}^{*} \cdot \boldsymbol{\eta} d \Gamma \quad \forall \boldsymbol{\eta} \in \mathcal{V}
$$

where $\mathcal{V}$ is the space of admissible variations of the displacements (virtual displacements), whose characteristic element has the following form:

$$
\boldsymbol{\eta}=\overline{\boldsymbol{\eta}}+H_{\mathcal{S}} \tilde{\boldsymbol{\eta}}
$$




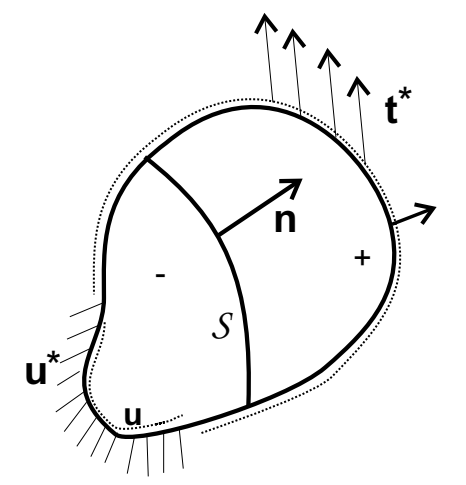

Figure 9. Boundary value problem.

$\mathbf{b}$ is the body force density vector and $\mathbf{t}^{*}$ is the traction vector prescribed on $\Gamma_{\boldsymbol{\sigma}}$.

After some mathematical treatment, it can be shown that the Euler-Lagrange equations that correspond to problem (19) if one considers (20) (see [33] for the proof) are

$$
\begin{array}{cc}
\nabla \cdot \boldsymbol{\sigma}+\mathbf{b}=\mathbf{0} \text { in } \Omega \backslash \mathcal{S} & \text { (momentum balance) } \\
\boldsymbol{\sigma} \cdot \boldsymbol{\nu}=\mathbf{t}^{*} \quad \text { on } \Gamma_{\boldsymbol{\sigma}} & \text { (prescribed tractions) } \\
\boldsymbol{\sigma}_{\Omega^{+}} \cdot \mathbf{n}-\boldsymbol{\sigma}_{\Omega^{-}} \cdot \mathbf{n}=\mathbf{0} \text { on } \mathcal{S} & \text { (outer traction continuity) } \\
\boldsymbol{\sigma}_{\Omega^{+}} \cdot \mathbf{n}-\boldsymbol{\sigma}_{\mathcal{S}} \cdot \mathbf{n}=\mathbf{0} \text { in } \mathcal{S} & \text { (inner traction continuity) }
\end{array}
$$

where $\boldsymbol{\nu}$ is the outward normal to $\partial \Omega$.

Now, let us consider the following viscous surface forces acting at $\mathcal{S}$ :

$$
\tilde{\mathbf{t}}=\bar{\gamma} \llbracket \dot{\mathbf{u}} \rrbracket \quad \text { in } \mathcal{S}
$$

where $\bar{\gamma}>0$ is a small viscosity-like parameter.

Now, let us propose the following viscosity-perturbed VBVP:

$$
\int_{\Omega} \nabla^{S} \boldsymbol{\eta}: \boldsymbol{\sigma} d \Omega=\int_{\Omega} \mathbf{b} \cdot \boldsymbol{\eta} d \Omega+\int_{\Gamma_{\sigma}} \mathbf{t}^{*} \cdot \boldsymbol{\eta} d \Gamma+\int_{\mathcal{S}} \underbrace{\bar{\gamma} \llbracket \dot{\mathbf{u}} \rrbracket}_{\tilde{\mathbf{t}}} \cdot \boldsymbol{\eta} d \Gamma \quad \forall \boldsymbol{\eta} \in \mathcal{V}
$$

Thus, the Euler-Lagrange equations corresponding to problem (26) are

$$
\begin{aligned}
\nabla \cdot \boldsymbol{\sigma}+\mathbf{b}=\mathbf{0} \text { in } \Omega \backslash \mathcal{S} & \text { (momentum balance) } \\
\boldsymbol{\sigma} \cdot \boldsymbol{\nu}=\mathbf{t}^{*} \quad \text { on } \Gamma_{\boldsymbol{\sigma}} & \text { (prescribed tractions) } \\
\boldsymbol{\sigma}_{\Omega^{-}} \cdot \mathbf{n}-\boldsymbol{\sigma}_{\Omega^{+}} \cdot \mathbf{n}=\bar{\gamma} \llbracket \dot{\mathbf{u}} \rrbracket \text { in } \mathcal{S} & \text { (perturbed outer traction continuity) } \\
\boldsymbol{\sigma}_{\mathcal{S}} \cdot \mathbf{n}-\boldsymbol{\sigma}_{\Omega^{+}} \cdot \mathbf{n}=\bar{\gamma} \llbracket \dot{\mathbf{u}} \rrbracket \text { in } \mathcal{S} & \text { (perturbed inner traction continuity) }
\end{aligned}
$$

From equations (26), and (30), it can be checked that the original equations (19), (23), and (24) are recovered as the damping-like parameter $\bar{\gamma}$ tends to zero. 
Uniqueness The main advantage of the perturbed problem (26) over its unperturbed version (19) comes from the uniqueness result for the displacement jump $\llbracket \mathbf{u} \rrbracket$ that we obtain here.

Let us consider that, for some time $t_{B}$ (the bifurcation time), two solutions of problem (26) in terms of the displacement jump emerge as bifurcated branches from the same fundamental branch. Let us denote the corresponding stress and rate of displacement jump fields as $\boldsymbol{\sigma}_{1}, \llbracket \dot{\mathbf{u}} \rrbracket_{1}$, for the first branch, and $\boldsymbol{\sigma}_{2}, \llbracket \dot{\mathbf{u}} \rrbracket_{2}$, for the second one. Let us also define $\Delta(\bullet):=(\bullet)_{2}-(\bullet)_{1}$. Then, since equation (26) has to hold for both solutions, substraction for both cases yields:

$$
\int_{\Omega} \nabla^{S} \boldsymbol{\eta}: \Delta \boldsymbol{\sigma} d \Omega=\int_{\mathcal{S}} \bar{\gamma} \Delta \llbracket \dot{\mathbf{u}} \rrbracket \cdot \boldsymbol{\eta} d \Gamma \quad \forall \boldsymbol{\eta} \in \mathcal{V} \text { for } t=t_{B}
$$

Since the bifurcation time $t_{B}$ is characterized by two solutions emerging from the same instantaneous values at the fundamental branch but with different evolutions: i.e.:

$$
\begin{gathered}
\Delta \llbracket \mathbf{u} \rrbracket=\Delta \boldsymbol{\sigma}=\Delta \varepsilon=\mathbf{0} \text { for } t=t_{B} \\
\Delta \llbracket \dot{\mathbf{u}} \rrbracket=\llbracket \dot{\mathbf{u}} \rrbracket_{2}-\llbracket \dot{\mathbf{u}} \rrbracket_{1} \neq \mathbf{0} \quad(a) \quad \text { for } t=t_{B} \\
\Delta \dot{\boldsymbol{\sigma}}=\dot{\boldsymbol{\sigma}}_{2}-\dot{\boldsymbol{\sigma}}_{1} \neq \mathbf{0}
\end{gathered}
$$

substitution into equation (31) reads

$$
\int_{\mathcal{S}} \bar{\gamma} \Delta \llbracket \dot{\mathbf{u}} \rrbracket \cdot \boldsymbol{\eta} d \Gamma=0 \quad \forall \boldsymbol{\eta} \in \mathcal{V}
$$

Consequently, since $\Delta \llbracket \dot{\mathbf{u}} \rrbracket \in \mathcal{V}$, taking $\boldsymbol{\eta}=\Delta \llbracket \dot{\mathbf{u}} \rrbracket$ equation (31) reads:

$$
\int_{\mathcal{S}} \bar{\gamma} \Delta \llbracket \dot{\mathbf{u}} \rrbracket \cdot \Delta \llbracket \dot{\mathbf{u}} \rrbracket d \Gamma=\int_{\mathcal{S}} \bar{\gamma}\|\Delta \llbracket \dot{\mathbf{u}} \rrbracket\|^{2} d \Gamma=0 \Rightarrow \Delta \llbracket \dot{\mathbf{u}} \rrbracket=\mathbf{0} \quad \text { for } t=t_{B} \text { and } \forall \mathbf{x} \in \mathcal{S}
$$

which contradicts equation (33)-(a). Therefore no bifurcation, in terms of the displacement jump produced by the crack arresting and activation, can occur for $\bar{\gamma}>0$. This states the beneficial effects of the viscous perturbation (25), on the uniqueness of the solution regardless of the size of the perturbing viscosity $\bar{\gamma}>0$.

6.2.2. Estimation of a critical time step The above uniqueness results guarantee that displacement jump bifurcation is avoided at the continuum level. However, in the context of a time advancing algorithm, it is expectable that the uniqueness of the discretized problem will depend on some critical value limiting the time step size (this can visualized in figure: 8). Then, all values of the time step size smaller than the critical time step will guarantee uniqueness of the time discretized problem. Here an estimate for this critical value is proposed.

When the so-called non-symmetric formulation of finite elements with elemental enrichment is used to discretize the rate form of a BVP corresponding to a solid undergoing strong discontinuities, the following expression in matrix form of the residual forces rate at time $t+\Delta t$ is obtained (see [28]):

$$
\underbrace{\left[\begin{array}{ll}
\mathbf{K}_{d d} & \mathbf{K}_{d \alpha} \\
\mathbf{K}_{\alpha d} & \mathbf{K}_{\alpha \alpha}
\end{array}\right]}_{\mathbf{K}_{t+\Delta t}} \underbrace{\left\{\begin{array}{c}
\dot{\mathbf{d}}_{t+\Delta t} \\
\dot{\boldsymbol{\alpha}}_{t+\Delta t}
\end{array}\right\}}_{\dot{\mathbf{a}}_{t+\Delta t}}=\underbrace{\left\{\begin{array}{c}
\dot{\mathbf{f}}_{t+\Delta t} \\
\mathbf{0}
\end{array}\right\}}_{\dot{\mathbf{F}}_{t+\Delta t}}
$$


where $\mathbf{d}_{t+\Delta t}$ is the vector containing the regular displacement degrees of freedom, $\boldsymbol{\alpha}_{t+\Delta t}$ is the vector having the degrees of freedom associated with the displacement jumps, and $\mathbf{f}_{t+\Delta t}$ is the external forces vector. In equation (36) $\mathbf{K}_{\alpha \alpha}$ has a diagonal-banded structure:

$$
\mathbf{K}_{\alpha \alpha}=\left[\begin{array}{ccc}
\cdot & \mathbf{0} & \mathbf{0} \\
\mathbf{0} & \mathbf{K}_{\alpha a}^{(e)} & \mathbf{0} \\
\mathbf{0} & \mathbf{0} & \cdot
\end{array}\right]
$$

where $\mathbf{K}_{\alpha a}^{(e)}$ stands for a $\left(n_{\mathrm{dim}} \times n_{\mathrm{dim}}, n_{\mathrm{dim}}=\right.$ problem dimension $)$ matrix corresponding to the enriching discontinuous modes in element $e$.

The addition of the viscous perturbation (25) leads to the following perturbed discrete problem:

$$
\begin{gathered}
\underbrace{\left[\begin{array}{cc}
\mathbf{K}_{d d} & \mathbf{K}_{d \alpha} \\
\mathbf{K}_{\alpha d} & \tilde{\mathbf{K}}_{\alpha \alpha}
\end{array}\right]}_{\tilde{\mathbf{K}}_{t+\Delta t}} \underbrace{\left\{\begin{array}{c}
\dot{\mathbf{d}}_{t+\Delta t} \\
\dot{\boldsymbol{\alpha}}_{t+\Delta t}
\end{array}\right\}}_{\dot{\mathbf{a}}_{t+\Delta t}}=\underbrace{\left\{\begin{array}{c}
\dot{\mathbf{f}}_{t+\Delta t} \\
\bar{\gamma} \dot{\boldsymbol{\alpha}}_{t}
\end{array}\right\}}_{\dot{\tilde{\mathbf{F}}}_{t+\Delta t}} \\
\tilde{\mathbf{K}}_{\alpha \alpha}=\left[\begin{array}{ccc}
\cdot & \mathbf{0} & \mathbf{0} \\
\mathbf{0} & \tilde{\mathbf{K}}_{\alpha a}^{(e)} & \mathbf{0} \\
\mathbf{0} & \mathbf{0} & \cdot
\end{array}\right] \quad \tilde{\mathbf{K}}_{\alpha a}^{(e)}=\mathbf{K}_{\alpha a}^{(e)}+\frac{\bar{\gamma}}{\Delta t} \mathbf{1}
\end{gathered}
$$

where 1 stands for the unit matrix. Uniqueness of the incremental discrete BVP is ensured if matrix $\tilde{\mathbf{K}}_{t+\Delta t}$ in (38) is positive definite. However, checking the positive definiteness of $\tilde{\mathbf{K}}_{t+\Delta t}$ represents a high computational burden. On the other hand, considering that $\mathbf{K}_{d d}$ is always positive definite, a way to assure positive definiteness of $\tilde{\mathbf{K}}_{t+\Delta t}$ consists of ensuring the positive definiteness of $\tilde{\mathbf{K}}_{\alpha \alpha}^{(e)}$ in equation (39) for every element $e$. Then, the problem of finding the critical time estimate $\Delta t^{\text {crit }}$ of (38) can be posed as follows:

$$
\begin{aligned}
& \text { FIND } \Delta t \text { such that } \\
& \hat{\mathbf{K}}_{\alpha \alpha}^{(e)}:=\mathbf{K}_{\alpha \alpha}^{(e)}+\frac{\bar{\gamma}}{\Delta t} \mathbf{1} \text { is positive definite } \forall e
\end{aligned}
$$

Considering that (Bromwich bounds) $\lambda_{\min }\left(\hat{\mathbf{K}}_{\alpha \alpha}^{(e)}\right)^{S} \leq \lambda\left(\hat{\mathbf{K}}_{\alpha \alpha}^{(e)}\right) \leq \lambda_{\max }\left(\hat{\mathbf{K}}_{\alpha \alpha}^{(e)}\right)^{S}$ where $\lambda_{\max }(\bullet), \lambda_{\min }(\bullet)$, and $\lambda(\bullet)$ are the maximum, the minimum, and an arbitrary eigenvalue of a square matrix $(\bullet)$, respectively, and that

$$
\lambda_{\min }\left[\left(\hat{\mathbf{K}}_{\alpha \alpha}^{(e)}\right)^{S}\right]=\lambda_{\min }\left[\left(\mathbf{K}_{\alpha \alpha}^{(e)}\right)^{S}\right]+\frac{\bar{\gamma}}{\Delta t} \geq 0 \quad \forall e
$$

Then, from equation (41), we can find a (conservative) solution for (40) as

$$
\Delta t^{\text {crit }}=\min _{e}\left(\frac{\bar{\gamma}}{\left\langle-\lambda_{\min }\left[\left(\mathbf{K}_{\alpha \alpha}^{(e)}\right)^{S}\right]\right\rangle}\right)
$$

where $\langle\bullet\rangle$ stands for the ramp function of $(\bullet)$. Equation $(42)$ entails the computation of eigenvalues of the small $\left(n_{\operatorname{dim}} \times n_{\operatorname{dim}}\right)$ matrix $\left(\mathbf{K}_{\alpha \alpha}^{(e)}\right)^{S}$. Therefore, computation of $\Delta t^{\text {crit }}$ is little time consuming. 

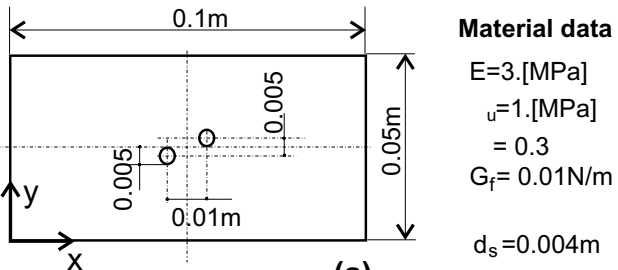

(a)

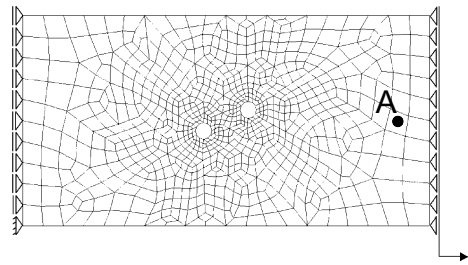

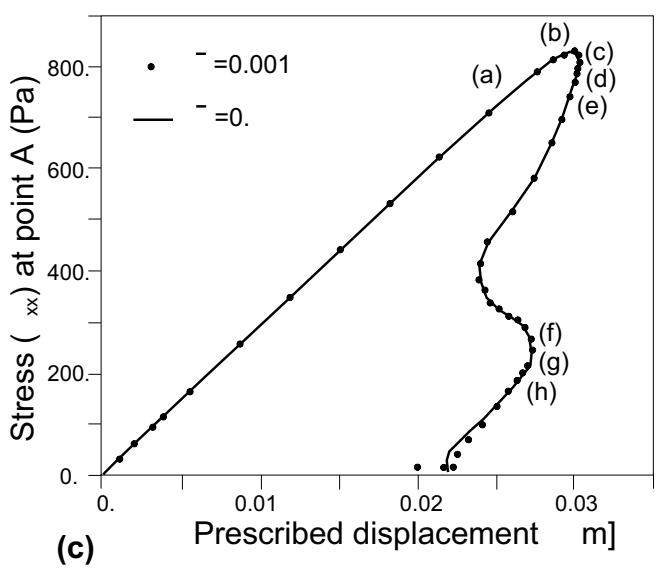

(c)

Figure 10. Plate with two geometrical imperfections: a: geometrical data; b: finite element mesh; c: stress at point $\mathrm{A}$ versus displacement $\delta$ indicating the loading levels at which pictures of the crack pattern (figure11) have been taken.

\section{NUMERICAL APPLICATIONS}

In this section representative examples of application of the CSDA are presented. Two of them correspond to multi-crack propagation problems in 2D. The third one is a $3 \mathrm{D}$ analysis of a well known test widely reported in the literature that has generally been solved twodimensionally by assuming plane stress conditions. With these examples, it is intended to show the potentiality of the strategies described in this paper. Although the first example could be run without the viscous perturbation strategy, it has proved essential in other examples, mainly in 3D problems. Similar observations can be made with respect to the shielding zone that was used with success in the first two examples where alternative strategies had failed.

An isotropic damage constitutive model, taken from reference [24], has been adopted in all cases as non-linear continuum material to model the continuum and discontinuum behaviour of concrete.

\subsection{Rectangular plate with two geometrical imperfections}

This test is inspired in a similar one presented in Diez et al. [10]. The dimensions of the specimen, boundary conditions and material data are shown in figure 10. In order to induce a strongly unstable behaviour a very brittle material was considered, by adopting a small value of the fracture energy $G_{f}$. Plane strain conditions have been considered.

Figure: 10-c plots the stress $\left(\sigma_{x x}\right)$ at point A (see figure: 10-b) versus the prescribed displacement $\delta$, with and without the artificial viscosity parameter $\bar{\gamma}$. It is clearly observed that small values of that parameter (whenever they are small) do not change the characteristic response.

The localization zones are triggered by the two openings representing structural imperfections. It is expected, from physical considerations, that the final failure mode 


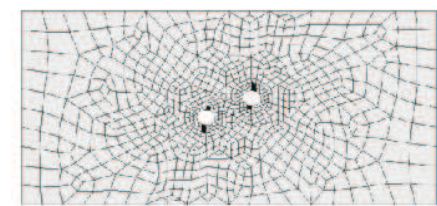

(a)

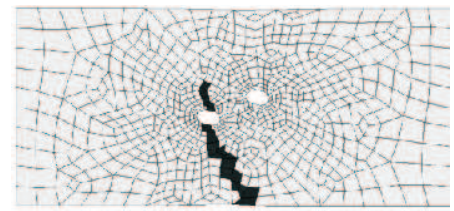

(d)

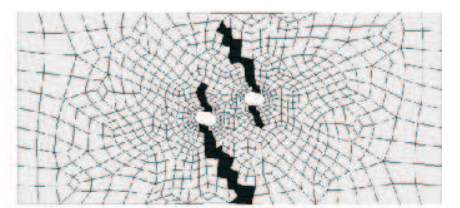

(b)

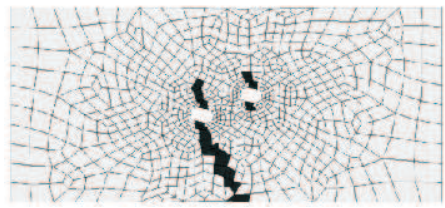

(c)

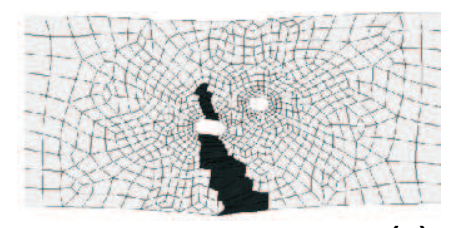

(e)

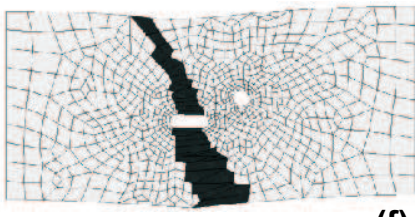

(f)

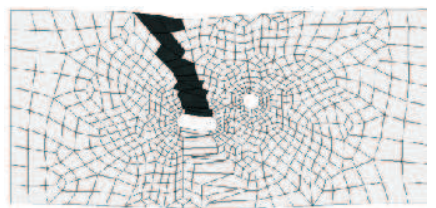

(g)

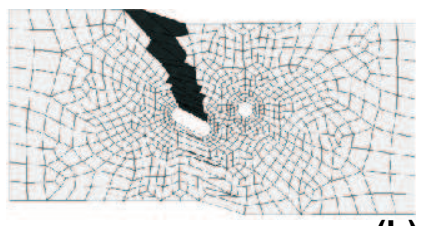

(h)

Figure 11. Plate with two geometrical imperfections: elements in inelastic loading (cracked elements) for increasing deformation stages.

corresponds to a unique active crack as shown in figure: 11-c:h. However, the fundamental equilibrium path shows two active symmetrical cracks, where the symmetry is a reflection with respect to the central plate point (see figure: 11-a:b). This symmetry is broken after crossing over a bifurcation point at the limit load (point (c) in figure 10-c) where one of the two cracks arrests.

\subsection{Mixed mode fracture of a double notched specimen}

The double-notched concrete specimen of figure 12 was tested by Nooru-Mohamed [20]. Numerical simulations have also been presented in [19] and [35]. Figure 12-a shows the geometry and applied loads. In order to induce a mixed mode of fracture the specimen was first loaded horizontally and then stretched vertically. The horizontal load was kept constant during the experimental test with a value $P_{h}=5 .[k N]$. Whereas, monotonically increasing displacements $\delta_{v}$ were imposed on the nodes at the top of the mesh.

For numerical modelling the considered material properties were: Young's modulus: $E=$ $3.2 \times 10^{10}[\mathrm{~Pa}]$, Poisson's ratio $\nu=0.2$, peak strength $\sigma_{u}=2.6 \times 10^{6}[\mathrm{~Pa}]$ and fracture energy $G_{f}=110 . \mathrm{N} / \mathrm{m}$. Plane stress conditions were adopted (thickness $t=0.05 \mathrm{~m}$ ), exclusion zone size $d_{S}=0.015 \mathrm{~m}$ was adopted and a perturbation viscosity $(\bar{\gamma}=0.001 \mathrm{Ns} / \mathrm{m})$ was added to help robustness of the iterative procedure.

The first (tensile) principal stress direction was used for determining the (orthogonal to it) crack propagation direction. The corresponding pattern of crack path candidates (the envelopes of the second principal stress field) are shown in figure: 12-b. The shielding zones are displayed in figure: 12 -c. 


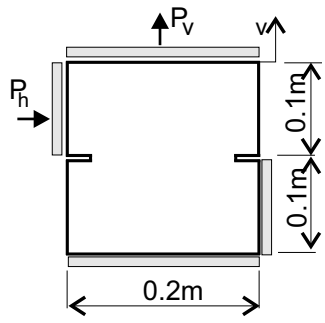

(a)

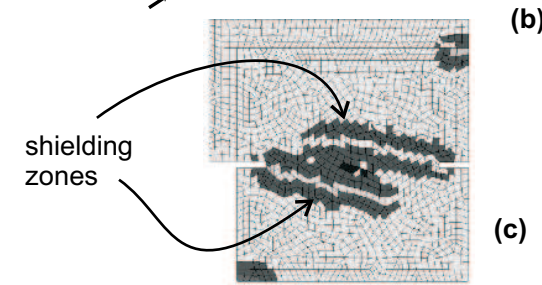

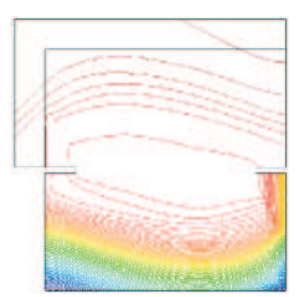

(b)

(c)

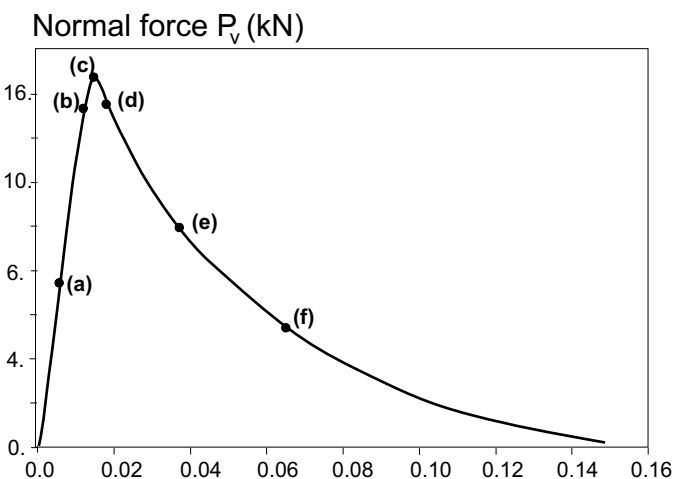

(d) Normal displacemen $\delta_{\mathrm{v}}\left(\times 10^{-3} \mathrm{~m}\right)$

Figure 12. Double notched specimen: a: geometry and loading system; b: crack path candidates (envelopes of the second principal stress field); c:shielding zones; d vertical load vs. displacement curve indicating the load levels corresponding to the cracking states of figure 13.

In figure: 13 a sequence of deformation and the evolution of several cracks are shown by means of pictures corresponding to the points in the curve of figure: 12-d. We notice again that the solution at early stages, including geometry and loading system, is symmetric with respect to the central point. However, the final solution displays a unique active (propagating) crack. Therefore, the same mechanism of breaking symmetry by means of a bifurcation procedure, already shown in the previous example takes place here.

\subsection{Four-point bending test}

From the original proposal by Arrea et al. [2] this test has been widely used to check numerical models in 2D cases (see for instance [31], [7] and [32]). Here a complete three-dimensional simulation in the context of the proposed CSDA is presented.

The geometry and loading conditions are shown in figure: 14-a:b. The material properties were: Young's modulus: $E=2.7 \times 10^{10}[\mathrm{~Pa}]$, Poisson's ratio $\nu=0.2$, peak strength $\sigma_{u}=$ 2. $\times 10^{6}[\mathrm{~Pa}]$, fracture energy $G_{f}=100 . \mathrm{N} / \mathrm{m}$., exclusion zone size $d_{S}=0.15 \mathrm{~m}$ and the perturbation viscosity $(\bar{\gamma}=0.01 \mathrm{Ns} / \mathrm{m})$. The deformed mesh is displayed in figure: 14-c and the load $P_{1}$ versus displacement point $\delta_{1}$ are plotted in 14-d. As commented above, the use of finite elements with elemental enrichment allows the elemental condensation of the additional discontinuity modes. This results into a moderate computational cost of the corresponding finite element analysis. As a matter of example, the three dimensional simulation allowing to trace the force-displacement curve in figure: 14-d, which was carried out in a personal computer equipped with an Intel/Pentium4 processor at 2,25 MHz. and 2Mb.of RAM, took, for a finite element mesh of 1681 tetrahedra and 1596 regular degrees of freedom, a CPU time of about 20 minutes. 


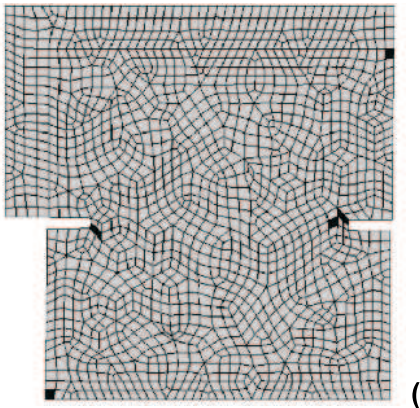

(a)

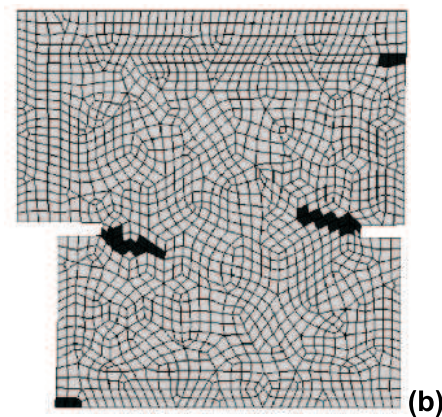

(b)
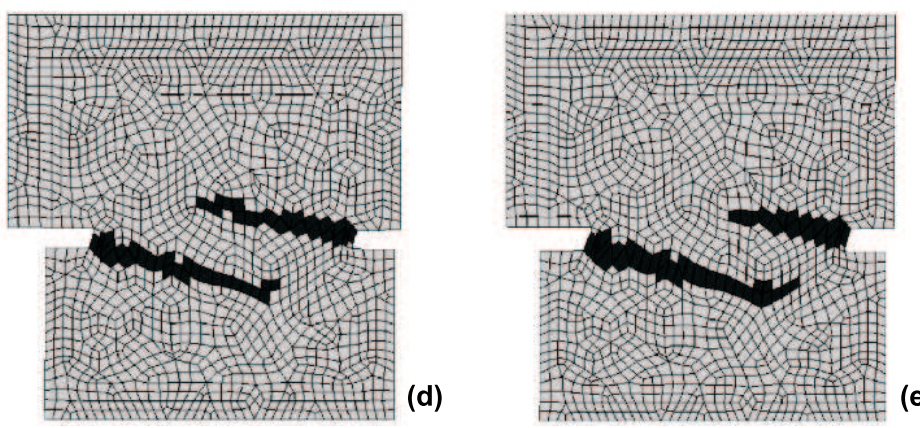

(e)

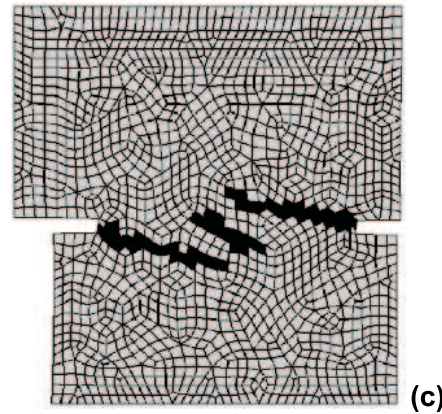

(c)

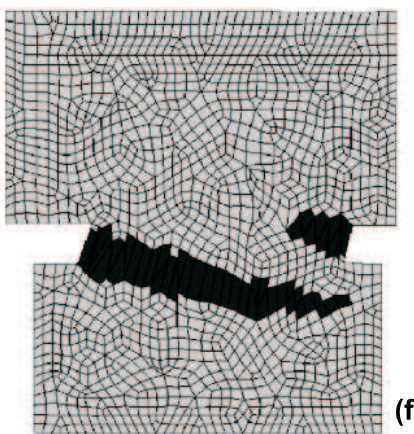

(f)

Figure 13. Mixed mode test: elements in inelastic loading for increasing deformation stages

\section{FINAL REMARKS}

Contributions to the numerical simulation of material failure in geomaterials in the context of the CSDA have been addressed in this work. Some final remarks on this novel aspects are given next:

- A heat conduction-like algorithm, to trace the crack paths, has been presented as an alternative to propagation techniques to track multiple strong discontinuities. It also allows and immediate extension from $2 \mathrm{D}$ to $3 \mathrm{D}$ cases. Indeed, since this algorithm accompanies the standard mechanical counterpart in the simulation process one could think of a cumbersome implementation and a significative increase of the computational costs. This is not the author's experience. Since the thermal-like problem is driven on the same finite element mesh than the mechanical one, no input heat flux is considered, and no boundary conditions other than the (trivial) adiabatic ones in the geometric boundary and two prescribed temperatures at the interior are imposed, many heavy implementation aspects are skipped. In essence, only the construction of the classical stiffness matrix for a heat conduction problem in equation (17) is the additionally required implementation. The resulting linear system can be either solved specifically or by a call to the solver of the used finite element package. Of course if that finite element package already allows for a thermal-mechanical problem, as the one used for this work [8], the implementation is almost trivial and only requires consideration of the specific conductivity tensor in 


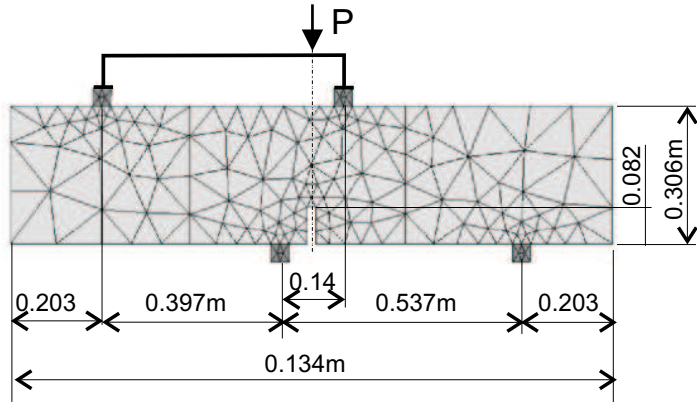

(a)

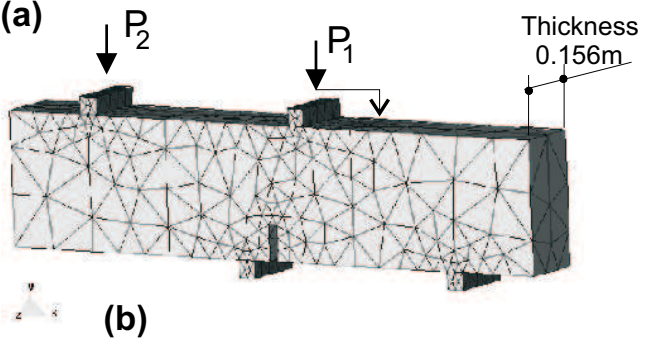

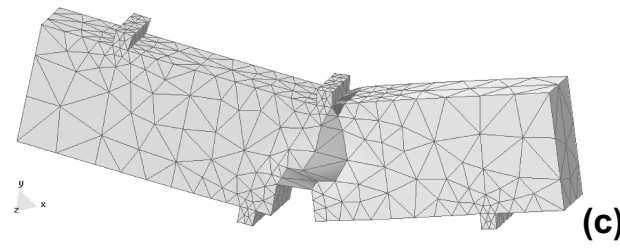

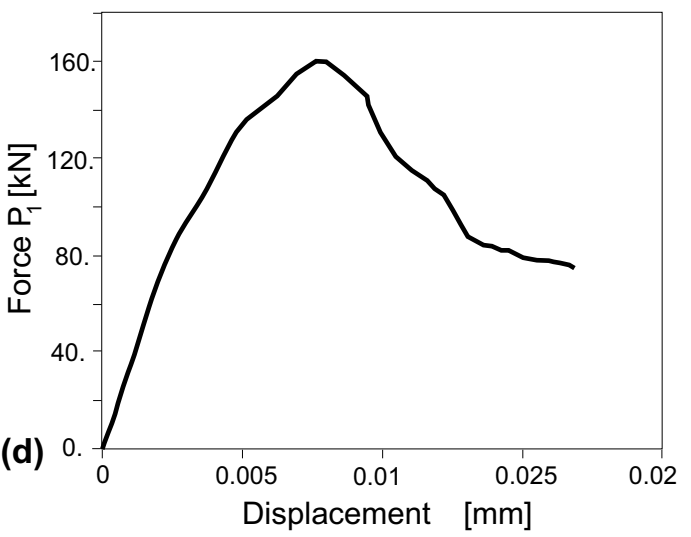

Figure 14. Four point bendig test: a) geometrical dimensions and loading system b) finite element mesh c) Deformed mesh d) load vs. displacement curve.

equation (15). As for the resulting computational costs, and since the involved problem is linear with only one degree of freedom per node and performed, at most, once every time step, they are almost negligible in front of the remaining costs of the traditional non-linear iterative procedure.

- From the author's experience, numerical simulations of multiple strong discontinuities exhibit, some times, spurious secondary cracking which do not necessarily come from a physical mechanism. The flatness of the stress distribution in the propagating crack tip neighborhood, in some cases, plus the numerical interpolation error induces, at some stages of the analysis, spurious cracking which causes troubling numerical responses. The shielding zone technique tries to increase robustness in front of this problem. In most of cases a shielding zone radius $d_{s}$ in equation (18) equivalent to the size of one or two elements is enough to prevent spurious secondary cracking.

- Multi-cracking problems introduce additional difficulties in the analysis. Combinations in terms of arrest and activation of the different cracks translate into bifurcation points in the equilibrium curve which should be resolved by some numerical procedure. In this paper, a technique to unfold the equilibrium path by introducing a viscous perturbation force acting on the discontinuity interfaces has been proposed. A critical time step is consequently derived to ensure algorithmic uniqueness in terms of the displacement jump. Though some times the proper load-response curve can be traced skipping that procedure, in most of the numerical simulations performed in this work on multi-cracking problems, the use of this technology has proved crucial, either to obtain physically sound numerical results or to increase robustness and, thus, decrease in a substantial manner 
the required computational time. The value of the perturbing viscosity $\bar{\gamma}$ in equation (30) has to be determined to keep in balance two aspects: 1) not to significantly change the structural response, mainly the dissipated energy, with respect to the same unperturbed problem and 2) not to decrease the critical time step $\Delta t^{\text {crit }}$ in equation (42) as to substantially increase the number of time steps necessary to solve the non-linear problem . Although more general studies should be performed for a wider range of problems, this seems to be a promising ingredient to include in numerical simulations of material failure.

\section{Acknowledgments}

Financial support from the Spanish Ministry of Science and Technology, through grant MAT2001-3863-C03-03, and from the Catalan Government Research Department, through the CIRIT grant 2001-SGR 00262, is gratefully acknowledged.

\section{REFERENCES}

1. F. Armero and K. Garikipati. An analysis of strong discontinuities in multiplicative finite strain plasticity and their relation with the numerical simulation of strain localization in solids. Int.J. Solids and Structures, 33(20-22):2863-2885, 1996.

2. M. Arrea and A.R. Ingraffea. Mixed-mode crack propagation in mortar and concrete. Technical report, 1982. Report 81-13, Dept. Struct. Eng., cornell Univ. New York.

3. Z.P. Bazant. Mechanics of distributed cracking. Appl. Mech. Rev, 39(5):675-701, 1986.

4. Z.P. Bazant and L. Cedolin. Stability of Structures. Oxford University Press, 1991.

5. T. Belytschko, N. Moës, S. Usui, and C. Parimi. Arbitrary discontinuities in finite elements. Int.J.Num.Meth.Engng., 50:993-1013, 2001.

6. P. Bocca, A. Carpintieri, and S. Valente. Size effect in the mixed mode crack propagation: softening and snap-back analysis. Engineering Fracture Mechanics, 35:159-170, 1990.

7. A. Carpintieri, S. Valente, G. Ferrara, and Melchiorri G. Is mode fracture energy a real material property? Computers \& Structures, 48(3):397-413, 1993.

8. M. Cervera, C. Agelet de Saracibar, and M. Chiumenti. COMET: a multipurpose finite element code for numerical analysis in solid mechanics. Technical report, Technical University of Catalonia (UPC), 2001.

9. E.W.V. Chaves. A three dimensional setting for strong discontinuities modelling in failure mechanics. Technical report, Doctoral Thesis, Dept. of Material Strength and Struct. in Eng., ETSCCP, Technical University of Barcelona, March, 2003.

10. P. Diez, M. Arroyo, and A. Huerta. Adaptivity based on error estimation for viscoplastic softening materials. Mechanics of Cohesive-frictional materials, 5:87-112, 2000.

11. MGD. Geers, R. de Borst, and RHJ. Peerlings. Damage and crack modelling in single-edge and doubleedge notched concrete beams. Eng. Fracture Mech., 65:247-261, 2000.

12. A. Hillerborg. Numerical methods to simulate softening and fracture of concrete. In G.C. Sih and A. Di Tomaso, editors, Fracture Mechanics of Concrete: Structural Application and Numerical Calculation, pages 141-170, 1985.

13. M. Jirasek. Comparative study on finite elements with embedded discontinuities. Computer Methods in Applied Mechanics and Engineering, 188:307-330, 2000.

14. M. Jirasek and T. Belytschko. Computational resolution of strong discontinuities. In H.A. Mang, F.G. Rammerstorfer, and J. Eberhardsteiner, editors, Proceedings of the Fifth World Congress on Computational Mechanics (WCCMV), page http://wccm.tuwien.ac.at. Vienna University of Technology, Austria, ISBN 3-9501554-0-6, July 7-12, 2002.

15. M. Jirasek and S. Rolshoven. Comparisson of integral-type nonlocal plasticity models for strain softening materials. Int. J. of Eng. Science, (Article in press, available online at www.scienciedirect.com), 2003.

16. R. Larsson, K. Runesson, and S. Sture. Embedded localization band in undrained soil based on regularized strong discontinuity theory and finite element analysis. Int.J. Solids and Structures, 33(20-22):3081-3101, 1996.

17. R. Larsson, P. Steinman, and K. Runesson. Finite element embedded localization band for finite strain plasticity based on a regularized strong discontinuity. Mechanics of Cohesive-Frictional Materials, 4:171194, 1998. 
18. N. Moës, J. Dolbow, and Belytschko T. A finite element method for crack growth without remeshing. Int.J.Num.Meth.Engng., 46:131-150, 1999.

19. W. Nechnech. Contribution a l'etude numerique du comportment du beton et des structures en beton arme soumis a des sollicitacions thermiques et mecaniques coupless. Technical report, Doctoral Thesis, Inst. Nat. des Sciences Appliques de Lyons, 2000.

20. N.B Nooru-Mohamed. Mixed-mode fracture of concrete: an experimental approach. Technical report, Ph.D. Thesis, Delft University of Technology, Delft, Netherlands, 1992.

21. Gastebled O.J. and May I.M. Bifurcation in the numerical solution of softening mechanisms. Computers and Structures, 78:745-755, 2000.

22. J. Oliver. Modeling strong discontinuities in solid mechanics via strain softening constitutive equations. Part 1: Fundamentals. Int.J.Num.Meth.Engng., 39(21):3575-3600, 1996.

23. J. Oliver. Modeling strong discontinuities in solid mechanics via strain softening constitutive equations. Part 2: Numerical simulation. Int.J.Num.Meth.Engng., 39(21):3601-3623, 1996.

24. J. Oliver. On the discrete constitutive models induced by strong discontinuity kinematics and continuum constitutive equations. International Journal Solids and Structures, 37:7207-7229, 2000.

25. J. Oliver, M. Cervera, and O. Manzoli. On the use of strain-softening models for the simulation of strong discontinuities in solids, in: Material Instabilities in Solids: Edited by R. de Borst and E. van der Giessen, chapter 8, pages 107-123. John Wiley \& Sons Ltd., 1998.

26. J. Oliver, M. Cervera, and O. Manzoli. Strong discontinuities and continuum plasticity models: The strong discontinuity approach. International Journal of Plasticity, 15(3):319-351, 1999.

27. J. Oliver, A. Huespe, M.D.G. Pulido, and E. Chaves. From continuum mechanics to fracture mechanics: the strong discontinuity approach. Engineering Fracture Mechanics, 69(2):113-136, 2002.

28. J. Oliver, A. Huespe, and E. Samaniego. A study on finite elements for capturing strong discontinuities. Int.J.Num.Meth.Engng., 56:2135-2161, 2003.

29. J. Oliver, A.E. Huespe, E. Samaniego, and E.W.V. Chaves. On strategies for tracking strong discontinuities in computational failure mechanics. In H.A. Mang, F.G. Rammerstorfer, and J. Eberhardsteiner, editors, Proceedings of the Fifth World Congress on Computational Mechanics (WCCM V), page http://wccm.tuwien.ac.at, Swansea, July 7-12, 2002. Vienna University of Technology, Austria, ISBN 3-9501554-0-6.

30. RHJ. Peerlings, R. de Borst, WAM. Brekelmans, and MGD. Geers. Gradient-enhanced damage modelling of concrete fracture. Mech. of Cohesive Frict. Mat., 3:323-342, 1998.

31. J. G. Rots. Computational Modeling of Concrete Fractures, Ph.D. Thesis. Delft University of Technology, 1988.

32. E. Samaniego. Contributions to the continuum modelling of strong discontinuities in two-dimensional solids. Technical report, Doctoral Thesis, Dept. of Material Strength and Struct. in Eng., ETSCCP, Technical University of Barcelona, March, 2003.

33. J. Simo and J. Oliver. A new approach to the analysis and simulation of strong discontinuities. In Z.P. Bazant et al., editor, Fracture and Damage in Quasi-brittle Structures, pages 25-39, 1994. E \& FN Spon.

34. J. Simo, J. Oliver, and F. Armero. An analysis of strong discontinuities induced by strain-softening in rate-independent inelastic solids. Computational Mechanics, 12:277-296, 1993.

35. B.W. Spencer. Finite elements with embedded discontinuities for modeling reinforced concrete members. Technical report, PhD. Thesis, Dept. Civil, Env. and Arch. Eng., University of Colorado, 2002.

36. M. Stolarska, D. L. Chopp, N. Moës, and T. Belytschko. Modelling crack growth by level sets in the extended finite element method. Int.J.Num.Meth.Engng., 51:943-960, 2001.

37. G.N. Wells and L.J. Sluys. A new method for modelling cohesive cracks using finite elements. Int.J.Num.Meth.Engng., 50:2667-2682, 2001.

38. K. Willam. Constitutive models for engineering materials. In Encyclopedia of Physical Science \& Technology (3rd edition), pages 603-633. Academic Press: New York, 2000.

39. O.C. Zienkiewicz and R.L. Taylor. The Finite Element Method. Butterworth-Heinemann, Oxford, UK, 2000 . 